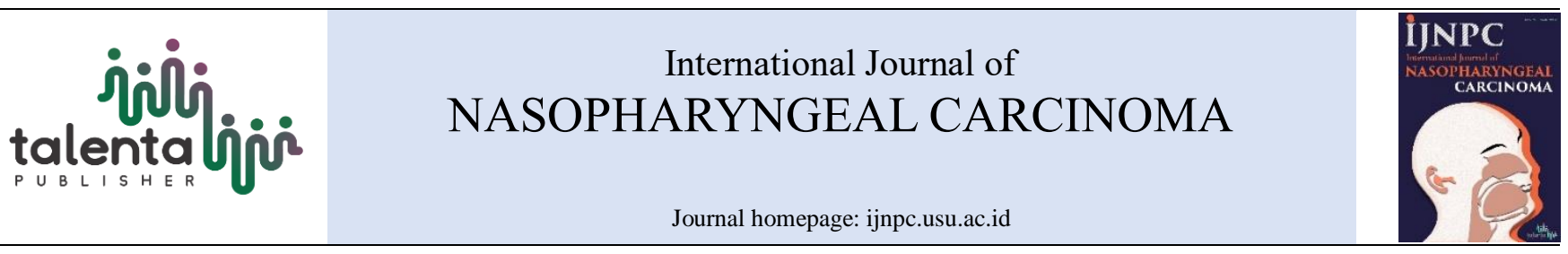

\title{
CORRELATION OF KI67 LEVEL TO METASTATIC EVENTS IN NASOPHARYNGEAL CARCINOMA
}

\author{
Eriza*, Ermalinda Kurnia, Denny Satria Utama \\ Department of Ear, Nose, Throat, Head-Neck Surgery, Faculty of Medicine Sriwijaya University/Dr. Mohammad Hoesin Palembang Hospital
}

\section{Abstract}

Introduction: Nasopharyngeal carcinoma (NPC) is a malignant squamous cell tumor occurring in the nasopharyngeal epithelial region where the cuboid epithelium is transformed into squamous cells. One of the typical signs of malignancy is uncontrolled cell proliferation. Ki67 is an immunohistochemical (IHC) test to assess cell proliferation activity. Increased Ki67 expression were associated with increased mitotic activity in cell proliferation.

Objective: To determine the correlation of Ki67 expression to regional and distant metastases events of nasopharyngeal carcinoma at Dr. Mohammad Hoesin Hospital Palembang.

Methods: This cross-sectional study was conducted on 31 subjects of NPC patients who had been diagnosed in Oncology Division of Department of Ear Nose and Throat Head Neck Surgery, then performed immunohistochemistry examination of Ki67 in Department of Anatomical Pathology of Dr. Mohammad Hoesin Hospital Palembang. The subjects were collected with consecutive sampling from October to December 2017 and performed other tests for staging such as computer tomography, chest x-ray and abdominal ultrasound.

Results: In the Ki67 level correlation analysis of the incidence of metastasis in nasopharyngeal carcinoma, the correlation coefficient was 0.98 with very strong correlation strength but the positive correlation was not statistically significant $(\mathrm{p}=0.599)$. However, in Ki67 level correlation analysis of histopathology result of nasopharyngeal carcinoma was found correlation coefficient of 0.385 and positive correlation which was statistically significant $(\mathrm{p}=0.032)$.

Conclusion: Ki67 levels of metastatic occurrence in nasopharyngeal carcinoma have no statistically significant correlation. However, Ki67 level on histopathological results of nasopharyngeal carcinoma and Ki67 immunoreactivity with primary tumor has a statistically significant correlation.
\end{abstract}

\section{Article Info}

Keywords:

Ki67 level, metastasis, nasopharyngeal carcinoma

\section{*Corresponding author:}

Address: Jl. Jendral Sudirman No. 2, Palembang, Sumatra Selatan.

e-mail: erizapatih@gmail.com

\section{INTRODUCTION}

Nasopharyngeal carcinoma (NPC) is a squamous cell malignant tumor that occurs in the border region of the nasopharyngeal epithelium where the cuboid epithelium changes into squamous epithelium. One typical sign of malignancy is uncontrolled cell proliferation. This is due to gene mutations that change the cellular metabolic system of tumor cells. Ki67 is one of an immunohistochemical examination (IHK) to assess cell proliferation activity. Ki67 protein is a kind of protein that located in the nucleus, useful for identification of cell proliferation. Ki67 is known as in indicator of mitosis, expressed in all phases of the cell cycle except the G0 phase. Increased expression of Ki67 is associated with increased of mitotic activity in cell proliferation [1-5].

NPC is included in top five malignant tumors with the highest frequency in Indonesia and in the head and neck department this was ranks first. NPC is ranked fourth after cervical cancer, breast cancer and skin cancer. The incident of NPC in Indonesia is around 6.3 per 100,000 population or around 12,000 new cases each year. At the Dr. Mohammad Hoesin Hospital Palembang from January 2013 until July 2017, there were 284 cases of nasopharyngeal carcinoma [6-10].

A study by Zhang et al. who sought for the association between Ki67 and $\mathrm{nm} 23$ expression with the clinicopathology of NPC patients in relation to the prognosis. In this study Ki67 expression was higher while $\mathrm{nm} 23$ was lower in the NPC group $(\mathrm{p}<0.01)$. Ki67 and nm23 expressions have correlation to disease course, clinical stage, and NPC metastasis. A study of Ozel et al. examined that the correlation between histological types and p53 and Ki67 expression in the NPC case with the result that there was a Ki67 expression that has directly correlation to the high expression of p53 [11-13].

$\mathrm{Hu}$ et al. detected cyclinD1 and Ki67 expression in NPC against its correlation with biological characteristic and prognosis. Patients with low cyclinD1 expression and high Ki67 expression have higher radio- sensitivity and have better prognosis. Ma et al., have analyzed the prognostic role of $\mathrm{p} 53$ oncoprotein, Ki67 antigen, tumor angiogenesis (Microvessel density-MVD), epidermal growth factor (EGFR) and HER2 receptor protein expression in Chinese population with undifferentiated NPC. The result was EGFR expression has a poor correlation with overall survival (OS) and disease-free survival (DFS) $(\mathrm{p}=0.0001$ dan $\mathrm{p} 0,01)$ and has a shorter time to progressive (TTP) $(\mathrm{p}=0.0001)$. There is no relationship between Ki67 and MVD with OS, DFS, TTP, and lymph node status. A study on correlation of Ki67 expression on regional and distant metastatic event in NPC has never been done before $[14,15]$. The purpose of this study was to determine the correlation of Ki67 expression on regional and distant metastatic events in NPC.

\section{MATERIAL AND METHODS}

This study was an observational analytic study with a cross-sectional design. Sample of this study were all patients with nasopharyngeal carcinoma in the ORL-HNS clinic in Dr. Mohammad Hoesin Hospital Palembang who met included in inclusion criteria from October 2017 until November 2017 that taken by consecutive sampling.

The inclusion criteria included patients with diagnosed of NPC who had complete medical record and documented detailed and accurately (interpretation of nasopharyngeal biopsy sourced from the anatomical pathology installation of dr. Mohammad Hoesin Hospital Palembang, computer tomography, chest X-ray and USG of the abdomen) and then determining the stage of nasopharyngeal carcinoma accordance with the AJCC 2010, while the sample of this study was excluded if the paraffin block was damaged so that IHK examination could not be performed to assess the level expression of Ki67.

The data of this study was collected into a study form that was prepared and then reported in tables and graphs. The collected data were statically 
analyzed in immunohistochemistry to assess the percentage of Ki67 expression in NPC patients. After that the data is processed using SPSS statistical data processing program, version 21.0. Univariate analysis was carried out on the data of the basic characteristics of research subjects to describe the research sample. To analyze the strength of correlation between the level of expression of Ki67 and tumor metastasis, a Pearson correlation test was performed if the data are normally distributed. If the data are not normally distributed or in the data are in categorical form, the Spearman correlation test is used. The $\mathrm{p}$ value is considered significant if $\mathrm{p}<0.05$ with a $95 \%$ confidence interval. Chi-square is used to see the relationship of confounding variables which in categorical. Data from statistical analysis results are reported in tables, graphs and by texture. Data processing and results will be assisted with SPSS software for windows version 21.0.

\section{RESULT}

This study was conducted on 31 patients with suspected nasopharyngeal carcinoma by anamnesis and physical examination and biopsy showed squamous cell carcinoma on nasopharynx. Based on gender distribution, 26 participants $(83.9 \%)$ were male and $5(16.1 \%)$ participants were female. The age data of the participants showed an abnormal distribution $(\mathrm{p}=0.045)$.

Distribution of participants based on age showed mean age 41.68 and median 43.00. This study shows that the youngest participants was 20 years old and the oldest participants was 58 years old.

Table 1. General characteristic (Study participants, $n=31$ )

\begin{tabular}{lrrccc}
\hline Variables & $\mathbf{N}$ & $\%$ & $\overline{\boldsymbol{X}} \pm$ SD & Median & $\begin{array}{c}\text { Normalit } \\
\text { y test }\end{array}$ \\
\hline Age (year) & & & $41.68 \pm 11.128$ & $43(20-58)$ & $\mathrm{P}=0.045$ \\
\hline Sex Male & 26 & 83.9 & & & \\
$\quad$ Female & 5 & 16.1 & & & \\
\hline BMI & 0 & 0 & $21.38 \pm 2.862$ & $20.4(17.9-26)$ & $\mathrm{P}=0.001$ \\
$\quad$ Very thin $(<17.0)$ & 5 & 16.1 & & & \\
$\quad$ Thin $(17.0-18.5)$ & 21 & 67.8 & & & \\
$\quad$ Normal $(18.5-25.0)$ & 5 & 16.1 & & & \\
$\quad$ Fat $(25.0-27,0)$ & 0 & 0 & & & \\
$\quad$ Very fat $(>27.0)$ & $\mathbf{3 1}$ & $\mathbf{1 0 0}$ & & & \\
\hline TOTAL & & & & & \\
\hline
\end{tabular}

$\mathrm{p}^{*}$ distribution normality test results based on the Shapiro-Wilk test; normally distributed if $\mathrm{p}>0.05$

Distribution based on body mass index (BMI) shows a median of 20.4 with the lowest BMI was 17.9 and the highest was 26.0. BMI participants were categorized as being very thin, thin, normal, fat, and very fat. The highest distribution of participants was in the normal category of $67.8 \%(21$ people). Then followed by the fat category for 5 participants $(16.1 \%)$ and the thin category in $16.1 \%$ ( 5 people)

Characteristics of Ki67 expression of this study can be seen in table 2 the highest Ki67 labelling index was at $51-75 \%$, with 11 participants (35.5\%). Then in $26-50 \%$ there were 9 participants (29\%). Labelling index $>75 \%$ for 8 participants $(25.8 \%)$. The least labelling index was $5-25 \%$ for 3 participants $(9.7 \%)$. Immunoreactivity $<4$ in 5 participants $(16.1 \%)$ and the most immunoreactivity was $\geq 4$ in 26 participants $(83.9 \%)$. The highes intensity of outward appearance was +2 in 15 participants $(48.4 \%)$ which the cytoplasm and membrane were smeared in yellow or dark brown. Furthermore, the intensity of outward appearance was +3 in 14 participants $(45.2 \%)$ which the cytoplasm and membrane were smeared in dark yellow or dark brown with an equal distribution. Intensity of +1 was found in 2 participants $(6.5 \%)$ which the cytoplasm and membrane were smeared with a faint yellow color. There is no cell which not smeared in yellow color.

The characteristic of the incidence of nasopharyngeal carcinomas in this study can be seen in table 3 . The metastatic score obtained a mean of 40.10 with a standard deviation of 19.03 , medium 52 with a minimum of 1 and maximum of 56 . The most common chief complain from participants was a lump in neck in 13 participants $(41.9 \%)$. Nasal complains such as nasal congestion and nosebleeds were felt in 11 participants $(35.5 \%)$. Headaches were felt in 5 participants $(16.1 \%)$. Double vision was complained in 1 participant (3.2\%) while the other symptoms of difficulty of swallowing were felt in 1 participant $(3.2 \%)$. The result of nasopharyngeal carcinoma biopsy in histopathology found squamous cell carcinoma (SCC) nonkeratinized in 3 participants $(9.7 \%$ ) and $\mathrm{SCC}$ undifferentiated in 28 participants $(90.3 \%)$. In this study there were no comorbidities other than nasopharyngeal carcinomas, such as diabetes mellitus, pulmonary tuberculosis, hypertension and immune deficiency diseases. In this study there were found Stage IV in 21 participants $(67.8 \%)$. Stage III was found in 8 participants $(25.8 \%)$. Stage I and stage II amounted to the same, were found in 1 participant $(3.2 \%)$. From this study, based on total clinical stage score showed an abnormal distribution which $\mathrm{p}=0.000$. The mean is 40.10 and median 52 on total clinical stage score.

Primary tumor $(\mathrm{T})$ obtained $\mathrm{T} 1$ in 1 participant $(3.2 \%)$ where tumor is limited to the nasopharynx. T2 found in 7 participants $(22.6 \%)$ where the tumor extends to the nasopharynx. T3 which the tumor was invading to bone structure and/or paranasal sinuses in 12 participants $(38.7 \%)$. T4 which tumor was expanding to intracranial and or involvement of cranial nerves, hypopharynx, orbital, or extending to the infratemporal fossa or masticator space, was found in 11 participants (35.5\%). Metastases in regional lymph nodes $(\mathrm{N})$, found the most common was $\mathrm{N} 2$ which occurred in bilateral lymph nodes metastases, the largest diameter was less than $6 \mathrm{~cm}$, above the supraclavicular fossa, in 13 participants $(41.9 \%)$. Followed by N3 in 12 participants (38.7\%) which lymph node metastases was greater than $6 \mathrm{~cm}$ and or extending to supraclavicular fossa. The absence of regional lymph node metastases was found in 4 participants (12.9\%). Unilateral lymph node metastases less than $6 \mathrm{~cm}(\mathrm{~N} 1)$ were found in 2 participants $(6.5 \%)$. Distant metastases (M1) were found in 9 participants (29\%) and 22 participants (71\%) were found no metastases (M0).

Table 2. Characteristic expression of KI67

\begin{tabular}{lrc}
\hline Variables & Total (N) & Persentage (\%) \\
\hline Labelling Index Ki67 & & \\
$5-25 \%$ & 3 & 9.7 \\
$26-50 \%$ & 9 & 29 \\
$51-75 \%$ & 11 & 35.5 \\
$\quad 75 \%$ & 8 & 25.8 \\
Imuno reactifity Ki67 & & \\
$<4$ & 5 & 16.1 \\
$\geq 4$ & 26 & 83.9 \\
Intensity of smeared & 0 & \\
0 & 2 & 0 \\
+1 & 15 & 6.5 \\
+2 & 14 & 45.4 \\
+3 & $\mathbf{3 1}$ & $\mathbf{1 0 0}$ \\
\hline Total & & \\
\hline
\end{tabular}

It is known that that the data is not distributed normally. Therefore, the correlation test between the level of $\mathrm{Ki} 67$ to the incidence of metastases in nasopharyngeal carcinoma, was carried out using the Spearman test. Correlation of Ki67 level to the incidence of metastases in nasopharyngeal carcinoma in Dr. Mohammad Hoesin Hospital Palembang did not have a significant correlation. However, the correlation of Ki67 levels with histopathological results of nasopharyngeal carcinoma and the correlation of Ki67 immunoreactivity to primary tumors in nasopharyngeal carcinoma have a significant correlation.

Table 3. Characteristic of nasopharyngeal events $(n=31)$

\begin{tabular}{|c|c|c|c|c|c|}
\hline \multicolumn{2}{|l|}{ Variables } & $\mathbf{N}$ & $\%$ & $\bar{X} \pm$ SD & Median \\
\hline \multicolumn{2}{|l|}{ Metastases score } & & \multicolumn{2}{|c|}{$40.10 \pm 19.03$} & $52(1-56)$ \\
\hline \multirow[t]{5}{*}{ Chief complains } & Lump in the neck & 13 & & 41.9 & \\
\hline & Nasal complains & 11 & & 35.5 & \\
\hline & Headache & 5 & & 16.1 & \\
\hline & Double vision & 1 & & 3.2 & \\
\hline & Others & 1 & & 3.2 & \\
\hline \multirow[t]{2}{*}{ Histophatology } & Nonkeratinized SCC & 3 & & 9.7 & \\
\hline & Undifferentiated SCC & 28 & & 90.3 & \\
\hline \multirow[t]{2}{*}{ Comorbidities } & $(+)$ & 0 & & 0 & \\
\hline & $(-)$ & 31 & & 100 & \\
\hline \multirow[t]{4}{*}{ Stadium } & I & 1 & & 3.2 & \\
\hline & II & 1 & & 3.2 & \\
\hline & III & 8 & & 25.8 & \\
\hline & IV & 21 & & 67.8 & \\
\hline \multirow[t]{4}{*}{$\mathrm{T}$} & T1 & 1 & & 3.2 & \\
\hline & $\mathrm{T} 2$ & 7 & & 22.6 & \\
\hline & T3 & 12 & & 38.7 & \\
\hline & $\mathrm{T} 4$ & 11 & & 35.5 & \\
\hline \multirow[t]{4}{*}{$\mathrm{N}$} & No & 4 & & 12.9 & \\
\hline & N1 & 2 & & 6.5 & \\
\hline & $\mathrm{N} 2$ & 13 & & 41.9 & \\
\hline & N3 & 12 & & 38.7 & \\
\hline \multirow[t]{2}{*}{ M } & M0 & 22 & & 71 & \\
\hline & M1 & 9 & & 29 & \\
\hline Total & & 31 & & 100 & \\
\hline
\end{tabular}

In the correlation analysis level of Ki67 to the occurrence of metastases in nasopharyngeal carcinoma obtained a correlation coefficient of 0.98 with very strong correlation and the positive correlation which not statistically significant $(\mathrm{p}=0.599)$. However, the correlation analysis level of KI67 to the 
histopathological results of nasopharyngeal carcinoma obtained a correlation coefficient of 0.385 with weak correlation strength and the positive correlation which statistically significant $(\mathrm{p}=0.0 .32)$.

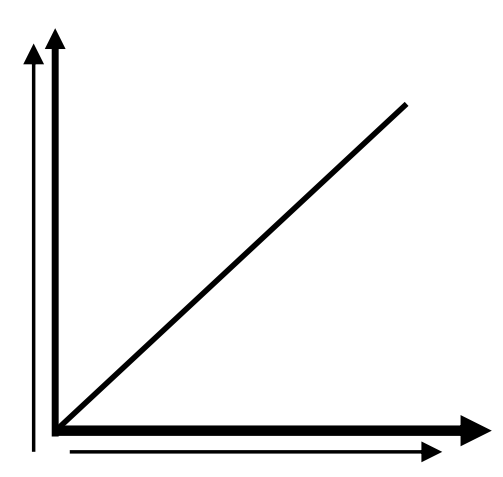

Metastases NPC

Graphic 1. Correlation between Level of KI67 to NPC metastases

Table 4. Correlation analysis between the level of KI67 to the result of metastases score in nasopharyngeal carcinoma

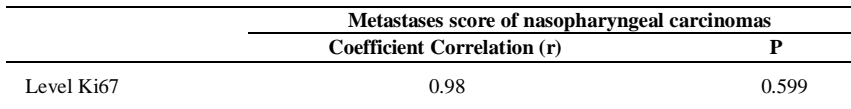

Table 5. Linear regression analysis of histopathological result in nasopharyngeal carcinoma

\begin{tabular}{lccc}
\hline & \multicolumn{3}{c}{ Histopathological nasopharyngeal carcinoma } \\
\cline { 2 - 4 } & B & R & Sig \\
\hline Level Ki67 & 1.22 & 0.385 & 0.032 \\
\hline
\end{tabular}

Table 6. Multivariate analysis factors associated to metastases of nasopharyngeal carcinoma

\begin{tabular}{rccc}
\hline Variabel & B & Sig & R \\
\hline Body mass index & 11.2 & 0.061 & 0.340
\end{tabular}

Multivariate analysis was performed to control for confounding variables, which used numerical data linear regression. Linear regression was performed level of Ki67 on metastatic scores in nasopharyngeal carcinoma. Metastases was more dominated by body mass index $(\mathrm{p}=0.61)$.

\section{DISCUSSION}

This is a cross sectional study that aims to determine the correlation between the level of Ki67 and the incidence of metastases in nasopharyngeal carcinoma in Dr. Mohammad Hoesin Hospital Palembang. This study was conducted at the Oncology Clinic Department of ORL-HNS Dr. Mohammad Hoesin Hospital Palembang. This study was conducted from October until December 2017. Sampling was done by consecutive sampling. About 31 patients, who treated with suspected nasopharyngeal carcinoma from anamnesis and physical examination, were performed biopsy. If the histopathological results of squamous cell carcinoma in nasopharynx, the clinical staging and immunohistochemical examination were performed. The number of samples that included in the criteria in this study were 31 participants. Based on sex distribution from this study, 26 participants $(83.9 \%)$ were male and $5(16.1 \%)$ participants were female. Data of the age showed an abnormal distribution $(\mathrm{p}=0.045)$. Distribution of participants based on age obtained mean age 41.68 and median 43.00 . In this study shows that the youngest participants were 20 years old and the oldest participants was 58 years old. Distribution based on body mass index (BMI) shows a median of 20.4 with the lowest BMI was 17.9 and the highest was 26.0. BMI participants were categorized as very thin, thin, normal, fat, and very fat. The highest distribution of participants was in the normal category of $67.8 \%$ (21 participants). Then followed by the fat category in 5 participants $(16.1 \%)$ and the thin category in $16.1 \%$ (5 participants)

From the study of Zhang et al (2016), from 59 participants they found 43 participants were male $(72.8 \%)$ and 16 participants were female $(27.2 \%)$, with the age between 15 to 80 years old, median 48.4 years old. Study by Ozel et al (2004) took samples from 20 paraffin blocks of nasopharyngeal carcinoma patients, 5 paraffin blocks were excluded due to damage. Out of the 15 paraffin blocks, 2 patients were female, and 13 patients were male. $\mathrm{Hu}$ et al. (2003) in their study reported 56 patients who participated in the study were 41 male and 15 females with the mean age was 49 years old (between 21 to 68 years old). In a study by Ma et al (2003), 78 participants were included in the study, of which 18 participants were female and 60 participants were male, with range of age between 22 to 79 years old and the median was 48 years old [12-15].

The most characteristic distribution of Ki67 expression was $51-75 \%$, in 11 participants $(35 \%)$. Then in $26-50 \%$ there were 9 participants $(29 \%)$. Labelling index $>75 \%$ in 8 participants $(25.8 \%)$. The least is $5-25 \%$ labelling in 3 participants $(9.7 \%)$. Immunoreactivity $<4$ was in 5 participants (16.1\%) and the most immunoreactivity was $\geq 4$ were in 26 participants (83.9\%). The highest intensity smeared was +2 in 15 participants $(48.4 \%)$ where the cytoplasm and membrane were smeared in yellow or dark brown. Furthermore, the intensity of smeared +3 was in 14 participants $(45.2 \%)$ where the cytoplasm and membrane were smeared in dark yellow or dark brown with an equal distribution. Smeared intensity of +1 was found in 2 participants $(6.5 \%)$ where the cytoplasm and membrane were smeared with a faint yellow color. There are no cells which not smeared in yellow color.

In a study of Zhang et al, they found that Ki67 was positive expressed in $4.35 \%(1 / 23)$ of nasopharyngeal mucosal tissue that experienced chronic inflammation and in $71.19 \%(45 / 59)$ of nasopharyngeal cancerous tissue. Ma et al. reported the results of their study that the expression of Ki67 was found in nasopharyngeal cancer. Low labelling index of 0-30\% in 18 patients $(25 \%)$, moderate labelling of $31-60 \%$ in 41 patients and high labelling index of $61-100 \%$ in 13 patients (18\%). According to Ozel et al. The expression of $\mathrm{Ki} 67$ was $11 \%$ for undifferentiated nasopharyngeal carcinoma, $27 \%$ for non-keratinized squamous cell carcinoma and $32 \%$ for differentiated squamous cell carcinoma. According to study $\mathrm{Hu}$ et al. Ki67 positive tumor cells from $0-31 \%$ reported in 40 patients $(71.4 \%)$ are low proliferated index and in 16 patients $(28.6 \%)$ are high proliferated index [12-15].

Distribution characteristics of the incidence of nasopharyngeal carcinoma in this study showed that the main complain felt by the patients was a lump in the neck in 13 participants $(41.9 \%)$. Nasal complains such as nasal congestion and nosebleeds were felt in 11 participants $(35.5 \%)$. Headaches were felt in 5 participants $(16.1 \%)$. Double vision was complained by 1 participant $(3.2 \%)$ while other symptoms difficulty of swallowing was felt in 1 participant $(3.2 \%)$. The result of histopathological biopsy of nasopharyngeal were squamous cell carcinoma (SCC) nonkeratinized in 3 participants $(9.7 \%)$ and SCC undifferentiated in 28 participants $(90.3 \%)$. In this study there were no comorbidities other than nasopharyngeal carcinomas such as diabetes mellitus, pulmonary tuberculosis, hypertension and immune deficiency disease. Stage IV mostly reported in this study, which found in 21 participants $(67.8 \%)$. Stage III was found in 8 participants $(25.8 \%)$. Stage I and II amounted the same, in 1 participant $(3.2 \%)$. From this study, based on total clinical stage showed abnormal distribution which $\mathrm{p}=0.000$. The mean was 40.10 and median was 52 on total clinical stage score.

Primary tumor (T) obtained T1 in 1 participant (3.2\%) which the tumor is limited in nasopharynx. T2 in 7 participants $(22.6 \%)$ which the tumor extends to the nasopharynx. T3 which the tumor was invading into bone structure and /or paranasal sinuses was found in 12 participants $(38.7 \%)$. T4 which the tumor has expansion to intracranial and or involvement of cranial nerves, hypopharynx, orbital, or extending to the infratemporal fossa or masticator space, was found in 11 participants (35.5\%). Metastases to regional lymph nodes $(\mathrm{N})$, mostly reported was $\mathrm{N} 2$ which occurred bilateral lymph nodes metastases, with the size less than $6 \mathrm{~cm}$, above supraclavicular fossa, was found in 13 participants $(41.9 \%)$. Followed by N3 found in 12 participants (38.7\%) which the size of metastases was greater than $6 \mathrm{~cm}$ and or extending to supraclavicular fossa. The absence of regional lymph nodes metastases was found in 4 participants $(12.9 \%)$. Unilateral lymph nodes metastases with the size less than $6 \mathrm{~cm}$ (N1) were found in 2 participants 
(6.5\%). Distant metastases (M1) were found in 9 participants $(29 \%)$ and in 22 participants $(71 \%)$ found that there were no metastases (M0).

The study conducted by Ma et al. reported that primary tumor T1 were found in 15 participants (19\%), T2 were in 33 participants $(42 \%)$, T3 were in 16 participants $(21 \%)$ and T4 were in 14 participants $(18 \%)$. Regional lymph node enlargement, N1 were found in 22 participants $(28 \%)$, N2 were in 15 participants $(19 \%)$, N3 were in 15 participants $(19 \%)$ whereas there were 26 participants $(33 \%)$ reported without enlargement of lymph node. The number of patients experiencing distant metastases was found in 7 participants $(8 \%)$. Those who were in stage I and II were 29 patients $(37 \%)$ while stage II and IV were 49 patients $(63 \%)$. According to the study of $\mathrm{Hu}$ et al reported histopathologic in 43 patients were poorly differentiated squamous cell carcinoma, 5 patients undifferentiated squamous cell carcinoma and 1 patient differentiated squamous cell carcinoma. The stage was determined based on the TNM classification of the Fuzhou conference in China in 1992 and found T1 in 12 patients. T2 in 22 patients, T3 in 18 patients and T4 in 4 patients. Stage I in 3 patients, stage II in 14 patients, stage III in 27 patients, and 12 patients in stage IV [14, 15].

Correlation level of $\mathrm{Ki} 67$ to the incidence of metastases in nasopharyngeal carcinoma in Dr. Mohammad Hoesin Hospital Palembang did not have a significant correlation. However, the correlation of Ki67 level in histopathological nasopharyngeal carcinoma and immunoreactivity of Ki67 in primary tumor in nasopharyngeal carcinoma have a significant correlation. Correlation analysis of Ki67 level to the occurrence of metastases in nasopharyngeal carcinoma reported the coefficient correlation was 0.98 with a very strong correlation and the positive correlation was not statistically significant $(\mathrm{p}=0.5999)$. However, in the linear regression analysis of $\mathrm{Ki67}$ level to the histopathological result of nasopharyngeal carcinoma, reported the coefficient correlation was 0.385 and the positive correlation was statistically significant $(p=0.032)$. Linear regression was performed on the Ki67 level to metastatic scores in nasopharyngeal carcinoma. Metastases was dominated by body mass index $(p=0.061)$.

According to the study of Zhang et al. the level of Ki67 was positive correlated to clinical stage and distant metastases. Patients who have a high $\mathrm{Ki} 67$ levels are found at the advanced stage (stage III/IV, $\mathrm{p}<0.05$ ). However, Ki67 expression was not significantly related to sex, age, cervical lymph node metastases and primary tumors. According to the study of Ozel et al., reported there was a correlation between histopathological subtypes of p53 and Ki67 expression in nasopharyngeal carcinoma. Several studies have reported a correlation between malignancy with the high level of Ki67 and its poor prognosis. However, other studies on prostate cancer and head-neck cancer, showed no correlation between Ki67 and its prognosis. According to study of Ma et al., there is no correlation between Ki67 and MVD on Overall Survival (OS), disease-free survival (DFS), progressive time and lymph node status. The study of $\mathrm{Hu}$ et al., reported patients with low cyclinD1 expression and high Ki67 expression have a higher radiosensitivity and better prognosis [12-15].

\section{CONCLUSION}

The conclusion was that the Ki67 labelling index was found mostly in $51-75 \%$ for 11 participants, followed by the second most was at $26-50 \%$ for 9 participants. The highest immunoreactivity of Ki67 was found $\geq 4$ in 26 participants. Smeared intensity was mostly found +2 in 15 participants. The level of Ki67 to the incidence of metastases in nasopharyngeal carcinoma has no statistically significant correlation. The level of Ki67 on histopathological results of nasopharyngeal carcinoma has a statistically significant correlation. Metastases is more dominated by body mass index and has a statistically significant correlation.

\section{REFERENCE}

[1] Wei W, Chua D. Nasopharyngal cancer. Dalam Bailey BJ, Healey GB, Johnson JT, Rosen CA dkk, penyunting. Head and neck surgeryotolaryngology Philadelphia Lippincott Williams \& Wilkins Edisi ke-4. 1875;97.

[2] Ma J, Cao S. The epidemiology of nasopharyngeal carcinoma. Nasopharyngeal Cancer: Springer; 2010. p. 1-7.

[3] Veer V. Current Diagnosis \& Treatment: Otolaryngology Head And Neck Surgery, 3rd edn. AK Lalwani, ed. McGraw-Hill Lange, 2011. ISBN 9780071624398 pp 978 Price 65.99 . The Journal of Laryngology \& Otology. 2012;126(9):974-.
[4] Roezin A, Adham M. Karsinoma Nasofaring, dalam Buku Ajar THT commit to user FKUI. Jakarta, hal; 2007.

[5] Adham M, Kurniawan AN, Muhtadi AI, Roezin A, Hermani B, Gondhowiardjo S, et al. Nasopharyngeal carcinoma in Indonesia: epidemiology, incidence, signs, and symptoms at presentation. Chinese journal of cancer. 2012;31(4):185.

[6] Ludman HS, Bradley PJ. ABC of ear, nose and throat: John Wiley \& Sons; 2012.

[7] Bansal M. Diseases of ear, nose and throat: JP Medical Ltd; 2012

[8] Kamran SC, Riaz N, Lee N. Nasopharyngeal carcinoma. Surgical Oncology Clinics. 2015;24(3):547-61.

[9] Tan L, Loh T. Benign and malignant tumors of the nasopharynx. Flint PW, Haughey BH, Lund VJ, Niparko JK, Richardson MA, Robbins KT Cummings Otolaryngology Head \& Neck Surgery 5th edition Philadelphia, PA: Mosby, Elsevier. 2010:1351-7.

[10] Feng Y-1, Xian W-j. The correlation between Ki67 immunoexpression before radiotherapy and $18 \mathrm{~F}-\mathrm{FDG} \mathrm{PET} / \mathrm{CT}$ values change in nasopharyngeal carcinoma during radiation: a pilot study. Journal of Nuclear Medicine. 2015;56(supplement 3):1293-

[11] Yang Y, Xuan J, Yang Z, Han A, Xing L, Yue J, et al. The expression of epidermal growth factor receptor and Ki67 in primary and relapse nasopharyngeal cancer: a micro-evidence for anti-EGFR targeted maintenance therapy. Medical Oncology. 2012;29(3):1448-55.

[12] Zhang J, Liu Y, Deng Y, He J, Lang J, Fan J. Ki67 and nm23 are potential prognostic markers in patients with nasopharyngeal carcinoma. Int J Clin Exp Pathol. 2016;9(6):6350-6.

[13] Özel E, Özbilim G, Dalmaz MG, Güney K. P53 and Ki-67 expression in nasopharyngeal carcinomas. Turkish Journal of Cancer. 2004;34(3):106-9.

[14] Hu G, Shi X. The Significance of the Expression of CyclinD1 and Ki67 Antigen in Nasopharyngeal Carcinoma. The Chinese-German Journal of Clinical Oncology. 2004;3(1):24-8.

[15] Ma BB, Poon TC, To K, Zee B, Mo FK, Chan CM, et al. Prognostic significance of tumor angiogenesis, $\mathrm{Ki} 67$, p53 oncoprotein, epidermal growth factor receptor and HER2 receptor protein expression in undifferentiated nasopharyngeal carcinoma-a prospective study. Head \& Neck: Journal for the Sciences and Specialties of the Head and Neck. 2003;25(10):864-72. 